\title{
Disjunctive questions, intonation, and highlighting ${ }^{\star}$
}

\author{
Floris Roelofsen and Sam van Gool \\ Amherst/Amsterdam
}

This paper examines how intonation affects the interpretation of disjunctive questions. The semantic effect of a question is taken to be three-fold. First, it raises an issue. In the tradition of inquisitive semantics, we model this by assuming that a question proposes several possible updates of the common ground (several possibilities for short) and invites other participants to help establish at least one of these updates. But apart from raising an issue, a question may also highlight and/or suggest certain possibilities, and intonation determines to a large extent which possibilities are highlighted/suggested. We will introduce a compositional version of inquisitive semantics, and extend this framework in order to capture the highlighting- and suggestion potential of sentences. This will lead to a systematic account of the answerhood conditions and implications of disjunctive questions with different intonation patterns.

\section{Preliminaries: basic assumptions and data}

Syntactic structure. Syntactically, we distinguish between two kinds of disjunctive interrogatives. On the one hand there are those that consist of a single interrogative clause containing a disjunction. On the other hand there are those that consist of two interrogative clauses, conjoined by disjunction. We will refer to the former as narrow-scope disjunctive interrogatives, and to the latter as wide-scope disjunctive interrogatives. Some examples are given in (1) and (2) below.

(1) Narrow-scope disjunctive interrogatives:

a. Does Ann or Bill play the piano?

b. Does Ann love Bill or Chris?

(2) Wide-scope disjunctive interrogatives:

a. Does Ann play the piano, or does Bill play the piano?

b. Does Ann play the piano, or Bill?

We will assume that (2b) has exactly the same underlying syntactic structure as (2a); only some material is left unpronounced.

Intonation patterns. Disjunctive questions can be pronounced in different ways, and their interpretation is partly determined by the choice of intonation pattern. We concentrate on two prosodic features that seem to have significant semantic impact. ${ }^{1}$ First, in the case of a narrow-scope disjunctive interrogative it is important whether the disjunction is pronounced 'as a block' or whether each of the disjuncts is given separate emphasis. Second, in case the disjuncts are given separate emphasis, it is important whether there is a rising or a falling pitch contour on the second disjunct. The different intonation patterns are given in (3) and (4), where underlining is used to represent emphasis, and $\uparrow$ and $\downarrow$ indicate rising and falling pitch. ${ }^{2}$

(3) Intonation patterns for narrow-scope disjunctive interrogatives:

a. Block intonation: Does Ann-or-Bill $\uparrow$ play the piano?

b. Open intonation: Does $\underline{\text { Ann } \uparrow}$ or Bill $\uparrow$ play the piano?

c. Closed intonation: Does $\underline{\text { Ann }} \uparrow$ or Bill $\downarrow$ play the piano?

\footnotetext{
^ This paper has benefited enormously from discussions with Ivano Ciardelli and Jeroen Groenendijk, for which we are very grateful. We would also like to thank Maria Aloni and Kathryn Pruitt for helpful feedback.

${ }^{1}$ The semantic significance of these prosodic features has been established experimentally by Pruitt (2007).

${ }^{2}$ Previous work on disjunctive questions usually distinguishes block intonation from closed intonation, but does not take the open intonation pattern into account (cf. Bartels, 1999; Han and Romero, 2004a,b; Beck and Kim, 2006).
} 
Intonation patterns for wide-scope disjunctive interrogatives:

a. Open intonation: Does Ann $\uparrow$ play the piano, or Bill $\uparrow$ ?

b. Closed intonation: Does $\underline{\text { Ann }} \uparrow$ play the piano, or Bill $\downarrow$ ?

Focus and closure. We take it that emphasis in the acoustic signal is a reflex of a focus feature in the logical form, and that the rising-and-falling pitch contour in (3c) and (4b) correlates with a closure feature in the logical form. It seems that this closure feature affects the pronunciation of the entire sentence (not just of, say, the contrastive elements in both disjuncts). Therefore, we assume that it is adjoined to the sentence as a whole. The ensuing logical forms are listed in the table below. Focus features, closure features, and interrogative complementizers are denoted by F, C, and Q, respectively.

\begin{tabular}{|c|c|c|}
\hline Pattern & Acoustic signal & Logical form \\
\hline $\begin{array}{l}\text { Narrow } \\
\text { - block } \\
\text { - open } \\
\text { - closed }\end{array}$ & 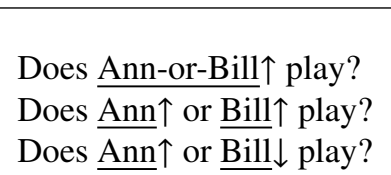 & $\begin{array}{l}\left.\text { [Q-does }[\text { Ann or Bill }]_{\mathrm{F}} \text { play }\right] \\
{\left[\mathrm{Q} \text {-does }[\text { Ann }]_{\mathrm{F}} \text { or }[\text { Bill }]_{\mathrm{F}} \text { play }\right]} \\
{\left[\mathrm{Q} \text {-does }[\text { Ann }]_{\mathrm{F}} \text { or }[\text { Bill }]_{\mathrm{F}} \text { play }\right]_{\mathrm{C}}}\end{array}$ \\
\hline $\begin{array}{l}\text { Wide } \\
\text { - open } \\
\text { - closed }\end{array}$ & $\begin{array}{l}\text { Does } \underline{\text { Ann }} \uparrow \text { play, or Bill } \uparrow ? \\
\text { Does } \underline{\text { Ann } \uparrow} \text { play, or } \underline{B i l l} \downarrow ?\end{array}$ & $\begin{array}{l}{\left[\left[\mathrm{Q} \text {-does }[\mathrm{Ann}]_{\mathrm{F}} \text { play }\right] \text { or }\left[\mathrm{Q} \text {-does }[\text { Bill }]_{\mathrm{F}} \text { play }\right]\right]} \\
{\left[\left[\mathrm{Q} \text {-does }[\text { Ann }]_{\mathrm{F}} \text { play }\right] \text { or }\left[\mathrm{Q} \text {-does }[\text { Bill }]_{\mathrm{F}} \text { play }\right]\right]_{\mathrm{C}}}\end{array}$ \\
\hline
\end{tabular}

Basic data. Our theory should capture, at the very least, the effects of intonation on answerhood conditions. The basic empirical observations are summed up in (5), (6), and (7) below (wide-scope disjunctive interrogatives are not explicitly listed here; they behave exactly like their narrow-scope counterparts in the relevant respects). Notice that open intonation behaves in some ways like block intonation, but in others more like closed intonation: it licenses a no answer, but it does not license a yes answer. To the best of our knowledge, this observation has not been taken into account before.
Does Ann-or-Billi play?
a. No. $\Rightarrow$ neither
b. $\quad$ Yes. $\Rightarrow$ at least one
c. (Yes,) Ann does.
d. (Yes,) Bill does.

(6)

Does $\underline{\text { Ann }} \uparrow$ or Bill $\uparrow$ play?

(7)

Does $\underline{\text { Ann }} \uparrow$ or Bill $\downarrow$ play?

a. No. $\Rightarrow$ neither

b. \#Yes. $\Rightarrow$ yes what?!

c. Ann does.

d. Bill does.
a. \#No.
b. \#Yes.
c. Ann does.
d. Bill does.

A further observation that should be accounted for is that disjunctive interrogatives with closure intonation convey that the speaker expects that exactly one of the disjuncts is true. In this respect, disjunctive interrogatives with closure intonation are similar to the corresponding disjunctive declaratives. However, there is also an important difference, as illustrated in (8) and (9):

(8) $\quad \underline{\text { Ann } \uparrow}$ or Bill $\downarrow$ plays the piano.

a. No, neither of them does.
(9) Does $\underline{A n n} \uparrow$ or Bill $\downarrow$ play the piano?

a. \#No, neither of them does.

b. Actually, neither of them does.

The difference is subtle but clear: (8) really excludes the possibility that neither Ann nor Bill plays, while (9) merely conveys an expectation on the speaker's part that at least one of them does. In the first case, disagreement can be expressed with no; in the second case, actually must be used instead.

The next section presents an analysis of disjunctive interrogatives in inquisitive semantics. This will not directly account for the above observations, but it will serve as a useful basis. 


\section{Inquisitive Semantics}

In inquisitive semantics, a sentence is taken to propose one or possibly several ways to update the common ground of a conversation. Formally, the proposition expressed by a sentence is a set of possibilities, each of which is in turn a set of indices, and represents a possible update of the common ground.

In previous work (Groenendijk, 2009; Mascarenhas, 2009; Groenendijk and Roelofsen, 2009; Ciardelli and Roelofsen, 2009; Ciardelli, 2009; Balogh, 2009, among others), inquisitive semantics has been defined for the language of propositional logic and the language of first-order predicate logic, largely abstracting away from issues of sub-sentential syntactic and semantic composition. In the present paper, we are specifically interested in this process of semantic composition at the sub-sentential level, and especially in the role that certain prosodic features play in that process. So, to start with, we need to define a compositional inquisitive semantics for a suitable fragment of English. Fortunately, much of the technical machinery that we need is familiar from alternative semantics (Hamblin, 1973; Kratzer and Shimoyama, 2002; AlonsoOvalle, 2006, among others).

Basic ingredients. As usual, we will say of each expression in our language that it is of a certain type. The basic types are $e, s$, and $t$, and whenever $\sigma$ and $\tau$ are types, $(\sigma \tau)$ is also a type. Our semantics will map each expression to a certain model-theoretic object. The type of an expression determines the kind of object that it is mapped to. Each model-theoretic object belongs to a certain domain. There is a domain $D_{e}$ of individuals, a domain $D_{s}$ of indices, and a domain $D_{t}$ consisting of the truth values 0 and 1 . Furthermore, for every complex type $(\sigma \tau)$ there is a domain $D_{(\sigma \tau)}$ consisting of all functions from $D_{\sigma}$ to $D_{\tau}$. As in alternative semantics, each expression of type $\tau$ is mapped to $a$ set of objects in $D_{\tau}$. The semantic value of an expression $\alpha$ will be denoted by $\llbracket \alpha \rrbracket$. Notice that $\llbracket \alpha \rrbracket$ is always a set. Therefore, we will refer to it as the denotation set of $\alpha$.

Semantic values are composed by means of pointwise function application:

Pointwise Function Application

$$
\begin{aligned}
& \text { If } \llbracket \alpha \rrbracket \subseteq D_{(\sigma \tau)} \text { and } \llbracket \beta \rrbracket \subseteq D_{\sigma} \text {, then } \\
& \llbracket \alpha \beta \rrbracket:=\llbracket \beta \alpha \rrbracket:=\left\{d \in D_{\tau} \mid \exists a \in \llbracket \alpha \rrbracket . \exists b \in \llbracket \beta \rrbracket, d=a(b)\right\}
\end{aligned}
$$

Basic lexicon. Most lexical items are mapped to singleton sets, consisting of their standard denotations.

$$
\begin{aligned}
& \text { a. } \llbracket A n n \rrbracket:=\{A n n\} \\
& \text { b. } \llbracket \text { Bill } \rrbracket:=\{\text { Bill }\} \\
& \text { c. } \quad \llbracket p l a y \rrbracket:=\left\{\lambda x \cdot \lambda w \cdot \text { play }_{w}(x)\right\} \\
& \text { d. } \llbracket \text { love } \rrbracket:=\left\{\lambda y \cdot \lambda x \cdot \lambda w \cdot \operatorname{love}_{w}(x, y)\right\}
\end{aligned}
$$

Disjunction. Disjunction introduces alternatives. The denotation set of a phrase ' $\alpha$ or $\beta$ ', where $\alpha$ and $\beta$ are two expressions of some type $\tau$, is the union of the denotation set of $\alpha$ and the denotation set of $\beta$ :

$$
\text { For any type } \tau \text {, if } \llbracket \alpha \rrbracket, \llbracket \beta \rrbracket \subseteq D_{\tau} \text {, then } \llbracket \alpha \text { or } \beta \rrbracket:=\llbracket \alpha \rrbracket \cup \llbracket \beta \rrbracket
$$

For example:

$$
\text { a. } \quad \llbracket A n n \text { or Bill } \rrbracket=\left\{\begin{array}{c}
\text { Ann, } \\
\text { Bill }
\end{array}\right\} \quad \text { b. } \llbracket \text { Ann or Bill plays } \rrbracket=\left\{\begin{array}{c}
\lambda w \cdot \text { play }_{w}(\text { Ann }), \\
\lambda w \cdot \operatorname{play}_{w}(\text { Bill })
\end{array}\right\}
$$

Notice that the denotation set of a complete sentence, such as 'Ann or Bill plays' is a set of objects in $D_{(s t)}$. Such objects are functions from indices to truth values, or equivalently, sets of indices. In inquisitive semantics, sets of indices are referred to as possibilities, and a set of possibilities is called a proposition. So complete sentences express propositions.

Visualization. As long as we limit our attention to a language that contains, besides disjunction, just two names, 'Ann' and 'Bill', and a single intransitive verb 'play', the propositions expressed by the sentences in our language can be visualized in a helpful way. For instance, the sentence 'Ann plays' expresses the

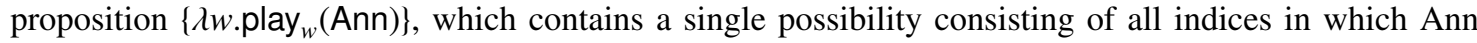
plays. This proposition is depicted in figure 1(a), where 11 is the index in which both Ann and Bill play, 10 


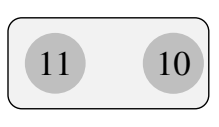

0100

(a) $P a$

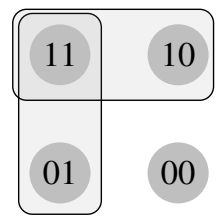

(b) $P a \vee P b$

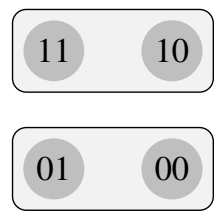

(c) $? P a$

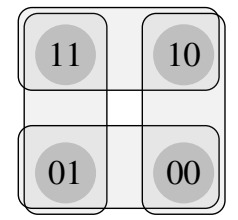

(d) $? P a \vee ? P b$

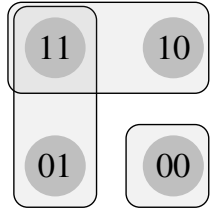

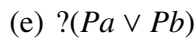

Fig. 1. Some propositions visualized.

the index in which only Ann plays, etcetera. Figure 1(b) depicts the proposition expressed by 'Ann or Bill plays'. As we saw in (13b), this proposition consists of two possibilities: the possibility that Ann plays, and the possibility that Bill plays.

Excluded possibilities. Recall that the possibilities for a sentence $\alpha$ embody the ways in which $\alpha$ proposes to update the common ground. If some index $i$ is not included in any possibility for $\alpha$, then we say that $i$ is excluded by $\alpha$. For in this case, $i$ will be eliminated from the common ground by any of the updates proposed by $\alpha$. If $\alpha$ excludes any indices, then we refer to the set of all such indices as the possibility excluded by $\alpha$. If $\alpha$ does not exclude any indices, then we say that it does not exclude any possibility. We use $[\backslash \alpha \downarrow]$ to denote the set of possibilities excluded by $\alpha$ (which is always either a singleton set, or empty).

Interrogative clauses. The interrogative complementizer, $\mathrm{Q}$, always operates on an expression $\alpha$ of type $(s t)$, and the resulting clause $[\mathrm{Q} \alpha]$ is always again of type $(s t)$. So even though there is a shift in syntactic category, there is no shift in semantic type. The proposition expressed by [Q $\alpha]$ consists of the possibilities for $\alpha$ itself, plus the possibility that $\alpha$ excludes.

$$
\llbracket \mathrm{Q} \alpha \rrbracket:=\llbracket \alpha \rrbracket \cup \llbracket \alpha \rrbracket
$$

For example, the proposition expressed by the simple polar interrogative 'Does Ann play?' consists of two possibilities: the possibility that Ann plays, and the possibility that she does not play. These possibilities embody two possible updates of the common ground, and the responder is invited to provide information such that either one of these updates can be established.

$$
\begin{aligned}
& \llbracket \text { Q-does Ann play } \rrbracket \\
= & \llbracket \text { Ann plays } \rrbracket \cup[] \text { Ann plays } \rrbracket=\left\{\begin{array}{c}
\lambda w \cdot \text { play }_{w}(\text { Ann }), \\
\lambda w \cdot \neg \text { play }_{w}(\text { Ann })
\end{array}\right\} \quad \Rightarrow \text { see figure } 1(\mathrm{c})
\end{aligned}
$$

Disjunctive interrogatives. Given these assumptions, the propositions expressed by wide- and narrowscope disjunctive interrogatives are the following:

$$
\begin{aligned}
& \text { Wide-scope disjunctive interrogative: Does Ann play or does Bill play? }
\end{aligned}
$$

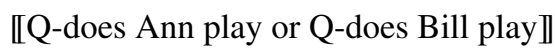

$$
\begin{aligned}
& =\llbracket \mathrm{Q} \text {-does Ann play } \rrbracket \cup \llbracket \mathrm{Q} \text {-does Bill play } \rrbracket \\
& =\left\{\begin{array}{c}
\lambda w \cdot \text { play }_{w}(\text { Ann }), \\
\lambda w \cdot \neg \text { play }_{w}(\text { Ann })
\end{array}\right\} \cup\left\{\begin{array}{c}
\lambda w \cdot \text { play }_{w}(\text { Bill }), \\
\lambda w \cdot \neg \text { play }_{w}(\text { Bill) }
\end{array}\right\} \\
& \Rightarrow \text { see figure } 1(\mathrm{~d})
\end{aligned}
$$

Narrow-scope disjunctive interrogative: Does Ann or Bill play?

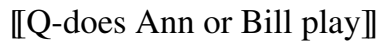

$$
\begin{aligned}
& =\llbracket \text { Ann or Bill plays } \rrbracket \cup[\rfloor \text { Ann or Bill plays }] \\
& =\left\{\begin{array}{c}
\lambda w \cdot \text { play }_{w}(\text { Ann }), \\
\lambda w \cdot \text { play }_{w}(\text { Bill })
\end{array}\right\} \cup\left\{\lambda w \cdot \neg \text { play }_{w}(\text { Ann }) \wedge \neg \text { play }_{w}(\text { Bill })\right\} \quad \Rightarrow \text { see figure } 1(\mathrm{e})
\end{aligned}
$$

So much for the compositional treatment of our basic fragment in inquisitive semantics. Notice that this treatment does not yet say anything about the licensing and interpretation of yes/no answers, or about 
the 'exactly one implication' of disjunctive interrogatives with closure intonation. The following sections propose an extension of the system that will allow us to capture these phenomena.

\section{Focus and highlighting}

The general idea that we would like to pursue in this section is that a sentence, besides proposing one or more possible updates, may also highlight certain possibilities, and that focus plays an important role in determining the possibilities that a sentence highlights.

We think that highlighting is of particular relevance for the licensing and interpretation of yes/no answers. More specifically, we hypothesize that a yes answer to a question $\alpha$ presupposes that $\alpha$ highlighted exactly one possibility, and if this presupposition is met, yes confirms that highlighted possibility. A no answer on the other hand, if felicitous, simply rejects all the possibilities highlighted by $\alpha$ (for now we will assume that a no answer is always felicitous; a felicity condition will be specified in section 4).

Initial motivation: opposing polar questions. Initial motivation for this idea comes from an old puzzle concerning polar questions, exemplified by the contrast between (18a) and (18b):
a. Is the door open?
b. Is the door closed?

According to inquisitive semantics, as it has been developed so far, (18a) and (18b) are equivalent: they both express a proposition consisting of two possibilities, the possibility that the door is open, and the possibility that the door is closed. However, there is a clear empirical difference between the two: in reply to (18a), yes means that the door is open, while in reply to (18b), it means that the door is closed. ${ }^{3}$

This difference is captured straightforwardly if we assume that (18a) highlights the possibility that the door is open, that (18b) highlights the possibility that the door is closed, and that the interpretation of yes and $n o$ is as hypothesized above. Our aim is to give a similar explanation of the licensing and interpretation of yes/no answers in response to disjunctive questions. In order to do so, we must first specify how the possibilities highlighted by a given sentence are compositionally determined, and in particular how focus affects this process.

Proposing and highlighting. We will henceforth assume that the semantic value of a sentence $\alpha$ consists of two components, $\llbracket \alpha \rrbracket_{\mathrm{P}}$ and $\llbracket \alpha \rrbracket_{\mathrm{H}}$. Both $\llbracket \alpha \rrbracket_{\mathrm{P}}$ and $\llbracket \alpha \rrbracket_{\mathrm{H}}$ are sets of possibilities; $\llbracket \alpha \rrbracket_{\mathrm{P}}$ embodies the proposal that $\alpha$ expresses, and $\llbracket \alpha \rrbracket_{\mathrm{H}}$ consists of the possibilities that $\alpha$ highlights.

The semantic value of subsentential expressions will also consist of these two components. For any expression $\alpha$, sentential or subsentential, we will refer to $\llbracket \alpha \rrbracket_{\mathrm{P}}$ as its P-set, and to $\llbracket \alpha \rrbracket_{\mathrm{H}}$ as its H-set. Both $\mathrm{P}$-sets and $\mathrm{H}$-sets are composed by means of pointwise function application.

What we used to call the denotation set of an expression, then, is now called its P-set. As far as names, verbs, and disjunction are concerned, $\mathrm{H}$-sets are defined just as P-sets. However, as soon as interrogative complementizers enter the derivation, $\mathrm{P}$-sets and $\mathrm{H}$-sets start to diverge. Recall that the proposal expressed by [Q $\alpha$ ] consists of the possibilities for $\alpha$ itself, plus the possibility that $\alpha$ excludes:

$$
\llbracket \mathrm{Q} \alpha \rrbracket_{\mathrm{P}}:=\llbracket \alpha \rrbracket_{\mathrm{P}} \cup[\downarrow \alpha \downarrow
$$

We will assume that $[\mathrm{Q} \alpha]$ simply highlights the possibilities that $\alpha$ itself highlights, not the possibility that $\alpha$ excludes:

$$
\llbracket \mathrm{Q} \alpha \rrbracket_{\mathrm{H}}:=\llbracket \alpha \rrbracket_{\mathrm{H}}
$$

These assumptions are sufficient to capture the contrast between opposing polar questions:

\footnotetext{
${ }^{3}$ This is sometimes taken to be a general argument against 'proposition set' approaches to questions-which include, besides inquisitive semantics, the classical theories of Hamblin (1973), Karttunen (1977), and Groenendijk and Stokhof (1984) — and in favor of alternatives such as the 'structured meaning' approach or the 'orthoalgebraic' approach (cf. Krifka, 2001; Blutner, 2009). Here, we choose not to pursue a full-fledged alternative to the proposition set approach, but rather to extend it in a suitable way.
} 
[Q-is the door open]

Proposes: open/closed

Highlights: open

yes $\Rightarrow$ the door is open

$n o \Rightarrow$ the door is closed
(22)

[Q-is the door closed]

Proposes: open/closed

Highlights: closed

yes $\Rightarrow$ the door is closed

$n o \Rightarrow$ the door is open

Highlighting and focus. We will assume that focus affects the computation of $\mathrm{H}$-sets. To see why, consider the two focus structures that give rise to block intonation and open intonation, respectively:
a. Does $[\text { Ann or Bill }]_{F}$ play the piano?
$\Rightarrow$ block intonation
b. Does $[A n n]_{F}$ or $[\text { Bill }]_{F}$ play the piano?
$\Rightarrow$ open intonation

Recall that (23a) licenses both yes and no as an answer, while (23b) only licenses no. Our hypothesis about the interpretation of yes and no captures this contrast if we assume that (23a) highlights a single possibility (the possibility that Ann or Bill plays), while (23b) highlights two possibilities (the possibility that Ann plays, and the possibility that Bill plays). But this can only be if focus affects the computation of $\mathrm{H}$-sets. For, apart from their focus structures, (23a) and (23b) are perfectly identical.

The intuitive idea that we will pursue is that 'focus makes H-sets collapse'. Let us first make this more precise for the case where $\alpha$ is a complete sentence, of type $(s t)$ :

$$
\begin{aligned}
& \text { If } \alpha \text { is of type (st), then: } \\
& \llbracket \alpha_{\mathrm{F}} \rrbracket_{\mathrm{H}}:=\left\{\bigcup_{\pi \in \llbracket \alpha \rrbracket_{\mathrm{H}}} \pi\right\}
\end{aligned}
$$

If $\alpha$ is of type (st), then every element of $\llbracket \alpha \rrbracket_{\mathrm{H}}$ is a possibility $\pi$, a set of indices. The focus feature collapses all these possibilities into one big possibility, $\bigcup_{\pi \in \llbracket \alpha \rrbracket_{\mathrm{H}}} \pi$. This, then, is the unique possibility in $\llbracket \alpha_{\mathrm{F}} \rrbracket_{\mathrm{H}} \cdot{ }^{4}$

If $\alpha$ is a sub-sentential expression, of some type $\sigma$ different from $(s t)$, then the elements of $\llbracket \alpha \rrbracket_{\mathrm{H}}$ are not full-fledged possibilities, so we cannot simply take their union. However, following Partee and Rooth (1982), we can take their 'generalized union':

$$
\begin{aligned}
& \text { If } \alpha \text { is of some type } \sigma \text {, different from (st), then: } \\
& \llbracket \alpha_{\mathrm{F}} \rrbracket_{\mathrm{H}}:=\left\{\lambda z \cdot \bigcup_{y \in \llbracket \alpha \rrbracket_{\mathrm{H}}} z(y)\right\} \quad \text { where } z \text { is a variable of type }(\sigma(s t))
\end{aligned}
$$

For our examples, the relevant case is the one where $\alpha$ is of type $e$. In this particular case, we have: ${ }^{5}$

$$
\llbracket \alpha_{\mathrm{F}} \rrbracket_{\mathrm{H}}:=\left\{\lambda P . \bigcup_{y \in \llbracket \alpha \rrbracket_{\mathrm{H}}} P(y)\right\} \quad \text { where } P \text { is a variable of type }(e(s t))
$$

Let us first consider what this means for some disjunctive declaratives with different focus structures:

$$
\begin{aligned}
& \mathbb{I}[\text { Ann }]_{\mathrm{F}} \text { or }[\text { Bill }]_{\mathrm{F}} \text { plays } \rrbracket_{\mathrm{H}}=\left\{\begin{array}{c}
\lambda w \cdot \operatorname{play}_{w}(\mathrm{Ann}), \\
\lambda w \cdot \operatorname{play}_{w}(\mathrm{Bill})
\end{array}\right\} \\
& \mathbb{I}[\text { Ann or Bill }]_{\mathrm{F}} \text { plays } \rrbracket_{\mathrm{H}}=\left\{\lambda w \cdot \operatorname{play}_{w}(\mathrm{Ann}) \cup \lambda w \cdot \text { play }_{w}(\text { Bill })\right\}
\end{aligned}
$$

With narrow focus on each individual disjunct, 'Ann or Bill plays' highlights two possibilities. But, as desired, focus on the whole disjunctive subject NP collapses these two possibilities into one. Now let us turn to disjunctive interrogatives. First consider the narrow-scope variant. Recall that, by definition, an interrogative clause $[\mathrm{Q} \alpha]$ highlights the same possibilities as $\alpha$ itself. So we have:

\footnotetext{
${ }^{4}$ Notice that this is reminiscent of what is called non-inquisitive closure in inquisitive semantics (cf. Groenendijk and Roelofsen, 2009), and what is called existential closure in alternative semantics (cf. Kratzer and Shimoyama, 2002).

${ }^{5}$ Computing the $\mathrm{H}$-set of a sentence with a focused expression of type $e$ in object position runs into type matching trouble in the present setup. The 'problem' is exactly the same as the one that arises for the interpretation of quantified noun phrases in object position in any system that starts with 'low types' (in particular, $(e(e t))$ for transitive verbs, cf. Heim and Kratzer, 1998). It also has the same solutions: type-lifting, function composition, quantifier raising, or simply starting out with higher types. For simplicity's sake, we will not implement any of these possible solutions here, and simply focus on examples with focused noun phrases in subject position.
} 


$$
\begin{aligned}
& \mathbb{I} \text { Q-does }[\text { Ann }]_{\mathrm{F}} \text { or }[\text { Bill }]_{\mathrm{F}} \text { play } \rrbracket_{\mathrm{H}}=\left\{\begin{array}{c}
\lambda w \cdot \operatorname{play}_{w}(\mathrm{Ann}), \\
\lambda w \cdot \operatorname{play}_{w}(\mathrm{Bill})
\end{array}\right\} \\
& \mathbb{I} \text { Q-does }[\text { Ann or Bill }]_{\mathrm{F}} \text { play } \rrbracket_{\mathrm{H}}=\left\{\lambda w \cdot \text { play }_{w}(\mathrm{Ann}) \cup \lambda w \cdot \text { play }_{w}(\text { Bill })\right\}
\end{aligned}
$$

Thus, it is predicted that the question 'Does Ann or Bill play?' only highlights two distinct possibilities if it has narrow focus on 'Ann' and on 'Bill'. Wide-scope disjunctive interrogatives on the other hand, always highlight two distinct possibilities:

$$
\mathbb{U} \text { Q-does }[\text { Ann }]_{\mathrm{F}} \text { play or Q-does }[\text { Bill }]_{\mathrm{F}} \text { play } \mathbb{H}_{\mathrm{H}}=\left\{\begin{array}{c}
\lambda w \cdot \text { play }_{w}(\text { Ann }), \\
\lambda w \cdot \text { play }_{w}(\text { Bill })
\end{array}\right\}
$$

The analysis so far yields a number of satisfactory predictions:

Does $[\text { Ann or Bill }]_{\mathrm{F}}$ play?

a. Highlights the possibility that Ann or Bill plays.

b. $y e s \Rightarrow$ at least one of them plays

c. $\quad n o \Rightarrow$ neither Ann nor Bill plays

(33) Does $[\text { Ann }]_{\mathrm{F}}$ or $[\text { Bill }]_{\mathrm{F}}$ play?

a. Highlights the possibility that Ann plays and the possibility that Bill plays.

b. yes $\Rightarrow$ presupposition failure (the question highlights more than one possibility)

c. $\quad n o \Rightarrow$ neither Ann nor Bill plays

(34) Does $[A n n]_{F}$ play or does $[\text { Bill }]_{\mathrm{F}}$ play?

a. Highlights the possibility that Ann plays and the possibility that Bill plays.

b. yes $\Rightarrow$ presupposition failure (the question highlights more than one possibility)

c. $\quad n o \Rightarrow$ neither Ann nor Bill plays

We seem to have obtained a better understanding of the basic difference between block intonation and open intonation. Now let us consider the effect of closure.

\section{Closure and suggestions}

Our basic intuition is that closure suggests that exactly one of the highlighted possibilities can be realized. (Recall that possibilities embody possible updates of the common ground; as such it makes sense to speak of them as 'being realized'.) To see what this amounts to, consider our running examples (35a) and (35b):

$$
\text { a. Does } \underline{A n n} \uparrow \text { or } \underline{B i l l} \downarrow \text { play the piano? } \quad \text { b. } \quad \text { Does } \underline{A n n} \uparrow \text { play the piano, or } \underline{B i l l} \downarrow \text { ? }
$$

These questions both highlight two possibilities: the possibility that Ann plays, and the possibility that Bill plays. To suggest that exactly one of these possibilities can be realized is to suggest that exactly one of Ann and Bill plays the piano. In particular, it is to suggest that at least one of them plays, and that they do not both play. Such a suggestion does indeed seem to be part of what (35a) and (35b) communicate.

There are several ways to formalize this intuition. We will assume here that the meaning of a sentence $\alpha$ does not just consist of $\llbracket \alpha \rrbracket_{\mathrm{P}}$ and $\llbracket \alpha \rrbracket_{\mathrm{H}}$, but has a third component, $\llbracket \alpha \rrbracket_{\mathrm{S}}$, which is the set of possibilities/updates that $\alpha$ suggests. We will refer to $\llbracket \alpha \rrbracket_{\mathrm{S}}$ as the S-set of $\alpha$.

We will assume that the S-set of expressions that do not bear a closure-feature is always empty, and define the semantic contribution of the closure-feature to be as follows:

$$
\text { The effect of closure: } \quad \llbracket \alpha_{\mathrm{C}} \rrbracket_{\mathrm{P}}:=\llbracket \alpha \rrbracket_{\mathrm{P}} \quad \llbracket \alpha_{\mathrm{C}} \rrbracket_{\mathrm{H}}:=\llbracket \alpha \rrbracket_{\mathrm{H}} \quad \llbracket\left[\alpha_{\mathrm{C}} \rrbracket_{\mathrm{S}}:=\mathcal{E} \mathcal{X}\left(\llbracket \alpha \rrbracket_{\mathrm{H}}\right)\right.
$$

The definition of $\llbracket \alpha_{\mathrm{C}} \rrbracket_{\mathrm{S}}$ makes use of the exclusive strengthening operator $\mathcal{E} X$. For any set of possibilities $\Pi$, and for any possibility $\pi \in \Pi$, the exclusive strengthening of $\pi$ relative to $\Pi$ is defined as:

$$
\mathcal{E} \mathcal{X}(\pi, \Pi):=\pi-\bigcup\{\rho \mid \rho \in \Pi \text { and } \pi \nsubseteq \rho\}
$$

and the exclusive strengthening of $\Pi$ itself is defined as: 


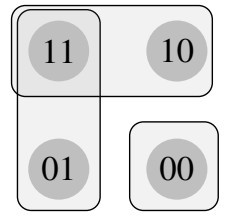

(a) $\llbracket(35 a) \rrbracket_{P}$

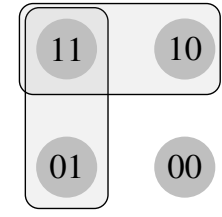

(b) $\llbracket(35 a) \rrbracket_{H}$

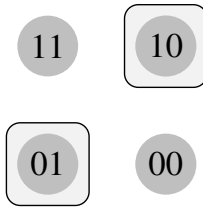

(c) $\llbracket(35 \mathrm{a}) \rrbracket_{\mathrm{S}}$

Fig. 2. Exclusive strengthening illustrated.

$$
\mathcal{E} \mathcal{X}(\Pi):=\{\mathcal{E} X(\pi, \Pi) \mid \pi \in \Pi\}
$$

The effect of exclusive strengthening is illustrated for example (35a) in figure 2. Recall that (35a) proposes three possibilities, as depicted in figure 2(a), and highlights two possibilities, as depicted in figure 2(b). Applying $\mathcal{E} \mathcal{X}$ to these two highlighted possibilities removes the overlap between them, resulting in the two possibilities in figure 2(c). This reflects the fact that (35a) suggests that exactly one of Ann and Bill plays the piano. The same result is obtained for (35b), since (35a) and (35b) highlight exactly the same possibilities. ${ }^{6}$

Accepting and canceling suggestions. Suggestions can either be accepted or canceled by a responder. We will assume that acceptance is the default. That is, if a suggestion is not explicitly contradicted, then all conversational participants assume that it is commonly accepted, and the suggested information is added to the common ground. Thus, if you ask (35a) or (35b), and I reply: 'Ann does', then I tacitly accept your suggestion. As a result, the common ground will not only be updated with the information that Ann plays, but also with the information that Bill does not play. ${ }^{7}$

Licensing no. At the beginning of section 3 we hypothesized that $n o$, in response to a question $\alpha$, simply denies all the possibilities that $\alpha$ highlights. We left the felicity condition on the use of $n o$ unspecified at that point. Now that suggestions have entered the picture, we are ready to make this felicity condition explicit.

Recall the contrast between disjunctive declaratives and interrogatives mentioned at the outset:

$\underline{\text { Ann } \uparrow}$ or Bill $\downarrow$ plays the piano.

a. No, neither of them does.

\section{Does $\underline{\text { Ann }} \uparrow$ or Bill $\downarrow$ play the piano?}

a. \#No, neither of them does.

b. Actually, neither of them does.

The declarative licenses a no response; the interrogative does not. What is the relevant difference between the two? - The answer is that the declarative really asserts that at least one of Ann and Bill plays the piano (in the sense that it excludes - technically speaking — the possibility that neither Ann nor Bill plays), whereas the interrogative merely suggests that at least one of Ann and Bill plays. Thus, this example illustrates that no can be used to deny an assertion, but not to cancel a suggestion. As illustrated in (40b), cancellation of a suggestion requires a 'weaker' disagreement particle such as actually or in fact (if a disagreement marker is used at all). ${ }^{8}$

Thus, $n o$, in response to a question $\alpha$, denies the possibilities that $\alpha$ highlights, but is felicitous only if denying these possibilities does not cancel the suggestion that $\alpha$ expresses. This accounts for the contrast between (39) and (40), and also for the licensing and interpretation of no in response to disjunctive interrogatives with block intonation or open intonation.

\footnotetext{
${ }^{6}$ It should perhaps be emphasized that closure is not interpreted here as signaling exhaustivity (as in Zimmermann, 2000). That is, it does not imply that 'nobody else plays the piano' or something of that kind. And this is for a good reason: disjunctive interrogatives with closure intonation generally do not exhibit any exhaustivity effects. Therefore, closure intonation and exhaustivity effects should be seen as (at least partly) independent phenomena.

${ }^{7}$ For reasons of space, we cannot spell out explicitly here how the common ground, and updates thereof, are modeled. Groenendijk (2008) and Balogh (2009) discuss the notion of a 'suggestion' that we make use of here in more detail, and provide formal definitions of acceptance and cancellation in the broader context of a dialogue management system.

${ }^{8}$ See (Groenendijk, 2008) and (Groenendijk and Roelofsen, 2009) for closely related observations.
} 
Sincerity requirements. Grice's (1975) quality maxim, formulated in our present terms, says that if a cooperative speaker $s$ utters a sentence $\alpha$, then $s$ must take himself to know that at least one of the updates proposed by $\alpha$ can indeed be established (informative sincerity). In inquisitive pragmatics (Groenendijk and Roelofsen, 2009), it is further assumed that if $\alpha$ is inquisitive, then for each update that $\alpha$ proposes, $s$ must be genuinely uncertain as to whether that update can indeed be established or not (inquisitive sincerity). In the present setting there is a third requirement, namely that if $\alpha$ suggests certain updates, then $s$ must genuinely expect that exactly one of these updates can indeed be established (expectative sincerity).

One consequence of this is that denying an assertion is much more likely to give rise to conflicts than canceling a suggestion. For, in the first case, the speaker's supposed knowledge is contradicted, while the second case may require merely a revision of expectations. This is illustrated by the following contrast:
A: $\underline{\text { Ann }} \uparrow$ or Bill $\downarrow$ is coming tonight.
$B$ : No, neither of them is.
A: What?! (\# Oh, thanks)
A: Is Ann $\uparrow$ or Bill $\downarrow$ coming tonight?
B: Actually, neither of them is.
A: Oh, thanks.

\section{Repercussions}

The proposed analysis may shed light on a much wider range of phenomena than the ones explicitly discussed here. Let us end by briefly mentioning some such phenomena:

Disjunctive declaratives. The analysis directly accounts for the 'exclusive component' of declarative disjunctions. In particular, it makes the right predictions for sentences like (43), which have received much attention in the recent literature (see Alonso-Ovalle, 2006, chapter 3, and references given there).

$\underline{\text { Ann } \uparrow}$ is coming, or Bill $\uparrow$, or both $\downarrow$.

Might. Ciardelli, Groenendijk, and Roelofsen (2009) provide an analysis of might in inquisitive semantics. Adopting this analysis, and assuming that a sentence might $\alpha$ highlights exactly the same possibilities as $\alpha$ itself, seems to give a satisfactory account of sentences like:
a. Jim might talk to Ann-or-Bill.
d. Jim might talk to $\underline{A n n} \uparrow$, or he might talk to Bill $\uparrow$.
b. Jim might talk to Ann $\uparrow$ or to Bill $\uparrow$.
e. Jim might talk to $\underline{A n n} \uparrow$, or he might talk to Bill $\downarrow$.
c. Jim might talk to $\underline{\text { Ann }} \uparrow$ or to $\underline{\text { Bill }} \downarrow$.
f. Jim might talk to $\underline{\operatorname{Ann}} \uparrow$, or to $\underline{B i l l} \uparrow$, or to both $\downarrow$.

Ignorance implicatures. Inquisitive pragmatics (in particular the inquisitive sincerity requirement mentioned above) accounts for ignorance implicatures triggered by disjunction, questions, and might in a uniform way. This account carries over straightforwardly to the extended semantic framework presented here.

Closure variability. One aspect of the data that we abstracted away from entirely is that the rising-andfalling pitch contour that was taken to signal closure may be pronounced more or less dramatically, and this seems to correlate with the strength of the corresponding 'exactly one' suggestion. This could be captured by construing the closure feature not as a binary-valued feature - that is either 'on' or 'off' - but rather as a continuous-valued feature-with values, say, between 0 and 1 . Phonologically, this value would then determine the sharpness of the rising-and-falling pitch contour, and semantically it would determine the strength of the corresponding 'exactly one' suggestion.

Cross-linguistic application. Of course, the syntactic structure and phonological characteristics of disjunctive questions differ widely across languages. However, the interpretation of disjunctive questions in different languages is usually reported to be similar or identical to the interpretation of their English counterparts. Therefore, we suspect that the general semantic mechanisms of proposing, highlighting, and suggesting possibilities may play a role cross-linguistically, even though the way in which these mechanisms are 'implemented' will differ from language to language. To give one example, it seems quite reasonable to hypothesize that while closure is signaled in English by intonation, it is expressed in other languages by designated lexical items. Haspelmath (2007) and Alonso-Ovalle (2006, chapter 5) provide data from Basque, Mandarin Chinese, Finnish, and several other languages that seems to support such a hypothesis. 


\section{Bibliography}

Alonso-Ovalle, L. (2006). Disjunction in Alternative Semantics. Ph.D. thesis, University of Massachusetts, Amherst.

Balogh, K. (2009). Theme with variations: a context-based analysis of focus. Ph.D. thesis, University of Amsterdam.

Bartels, C. (1999). The intonation of English statements and questions: a compositional interpretation. Routledge.

Beck, S. and Kim, S. (2006). Intervention effects in alternative questions. The Journal of Comparative Germanic Linguistics, 9(3), 165-208.

Blutner, R. (2009). Questions and answers in an orthoalgebraic approach. Manuscript, University of Amsterdam, available via www. blutner. de.

Ciardelli, I. (2009). Inquisitive semantics and intermediate logics. Master Thesis, University of Amsterdam.

Ciardelli, I. and Roelofsen, F. (2009). Inquisitive logic. To appear in the Journal of Philosophical Logic, available via www.illc.uva.nl/inquisitive-semantics.

Ciardelli, I., Groenendijk, J., and Roelofsen, F. (2009). Attention! Might in inquisitive semantics. In S. Ito and E. Cormany, editors, Proceedings of Semantics and Linguistic Theory (SALT XIX).

Grice, H. (1975). Logic and conversation. In P. Cole and J. Morgan, editors, Syntax and Semantics, volume 3, pages 41-58.

Groenendijk, J. (2008). Inquisitive semantics and dialogue pragmatics. Rutgers lecture notes, available via www.illc.uva.nl/inquisitive-semantics.

Groenendijk, J. (2009). Inquisitive semantics: Two possibilities for disjunction. In P. Bosch, D. Gabelaia, and J. Lang, editors, Seventh International Tbilisi Symposium on Language, Logic, and Computation. Springer-Verlag.

Groenendijk, J. and Roelofsen, F. (2009). Inquisitive semantics and pragmatics. Presented at the Workshop on Language, Communication, and Rational Agency at Stanford, available via www.illc.uva. nl/inquisitive-semantics.

Groenendijk, J. and Stokhof, M. (1984). Studies on the Semantics of Questions and the Pragmatics of Answers. Ph.D. thesis, University of Amsterdam.

Hamblin, C. L. (1973). Questions in Montague English. Foundations of Language, 10, 41-53.

Han, C. and Romero, M. (2004a). Disjunction, focus, and scope. Linguistic Inquiry, 35(2), 179-217.

Han, C. and Romero, M. (2004b). The syntax of whether/Q... or questions: Ellipsis combined with movement. Natural Language $\mathcal{E}$ Linguistic Theory, 22(3), 527-564.

Haspelmath, M. (2007). Coordination. In T. Shopen, editor, Language typology and syntactic description, volume II: complex constructions, pages 1-51. Cambridge University Press.

Heim, I. and Kratzer, A. (1998). Semantics in Generative Grammar. Blackwell Publishers.

Karttunen, L. (1977). Syntax and semantics of questions. Linguistics and Philosophy, 1, 3-44.

Kratzer, A. and Shimoyama, J. (2002). Indeterminate pronouns: The view from Japanese. In Y. Otsu, editor, The Proceedings of the Third Tokyo Conference on Psycholinguistics, pages 1-25.

Krifka, M. (2001). For a structured meaning account of questions and answers. Audiatur Vox Sapientia. A Festschrift for Arnim von Stechow, pages 287-319.

Mascarenhas, S. (2009). Inquisitive semantics and logic. Master Thesis, University of Amsterdam.

Partee, B. H. and Rooth, M. (1982). Generalized conjunction and type ambiguity. In A. von Stechow, editor, Meaning, Use, and Interpretation. de Gruyter.

Pruitt, K. (2007). Perceptual relevance of prosodic features in non-wh-questions with disjuction. Manuscript, University of Massachusetts Amherst.

Zimmermann, E. (2000). Free choice disjunction and epistemic possibility. Natural Language Semantics, 8, 255-290. 